\title{
LA TEORÍA CLÁSICA DEL DON Y LA DONACIÓN DE SANGRE ${ }^{1}$
}

\author{
DAVID CASADO NEIRA \\ Universidad de Vigo
}

\author{
PALABRAS CLAVE ADICIONALES \\ ADDITIONAL KEYWORDS \\ Transfusión, Intercambio, Biomedicina, Regalo, Transfusion, Exchange, Biomedicine, "Gift, \\ Donante. \\ Donor.
}

RESUMEN. La teoria del don enunciada por Marcel Mauss es en esta ocasión utilizada para analizar la donación de sangre (voluntaria, no remunerada y universal). Las tres obligaciones que implica el don (dar, recibir y devolver) no están ausentes en la moderna donación de sangre, así como tampoco lo está la vigencia del don como forma de intercambio social; pero en la transfusión de sangre el don adquiere unas características específicas que, si bien no nos permite considerar esta donación como una forma convencional de don, tampoco es posible obviar su vigencia. Parte de los problemas y conflictos generados en torno a esta práctica biomédica no pueden ser comprendidos si no se presta atención a la difusa persistencia del don en contextos sociales fuertemente institucionalizados.

ABSTRACT. The Gift Theory exposed by Marcel Mauss is here used to analyze free, not paid and universal blood donation. According to Mauss, every gift involves three obligations: to give, to get and to return. All of them are present in modern blood donation, even gift is still in force as social exchange. But the gift of blood acquires some specific characteristics and, even when it does not follow the conventional patterns of a gift, it is not possible to obviate its validity. Some problems and conflicts generated under this biomedical practice would be understood if attention to the fuzzy persistence of the logic of gift were played, also in strongly institutionalized social contexts.

\footnotetext{
${ }^{1} \mathrm{La}$ investigación sobre la que se sustenta este texto fue realizada en Galicia entre los años 1993 y 2001, a raíz de la puesta en marcha de un nuevo banco de sangre central para toda la Comunidad Autónoma (Centro de Transfusión de Galicia). Toda la información originalmente en gallego ha sido traducida aquí.

E-mail: dcneira@uvigo.es
}

Revista Internacional de Sociología (RIS)

Tercera Época, nº 34, Enero-Abril, pp. 107-133, 2003. 
RIS

REVISTA INTERNACIONAL DE SOCIOLOGIA

№ 34, Enero-Abril, 2003

DAVID CASADO NEIRA

La donación de sangre, conforme a la legislación vigente, se define como un acto voluntario, no remunerado y universal, es decir, como un tipo muy específico de regalo hecho a alguien desconocido. El sistema actual de hemodonación se desarrolla a partir del Real Decreto 1854/1993, 22 de octubre de 1993, del Ministerio de Sanidad y Consumo, por el que se establecen los requisitos técnicos y las condiciones mínimas de la hemodonación y de los bancos de sangre. Esta manifestación moderna de altruismo supone la constitución de una forma de don que, si bien poco tiene que ver con otras formas de regalar, sí está a la vez inmersa en dinámicas inherentes a las maneras en las que emerge la reciprocidad, como todo lo que circula en el seno de una comunidad.

Lo dado como actante social, como entidad que actúa en el sistema social, provoca una lógica ${ }^{2}$ propia que se manifiesta en cómo circula: lo que se da es medio y fin de reciprocidad, pero también determina ésta. Mi propuesta ês indagar en la realización del acto de donar en sus aspectos estructurales, no como un acto de decisión que emerge soberanamente de la consciencia del sujeto, sino recurriendo, para ello, a la teoría clásica del don que Marcel Mauss enunció en su bello Essai sur le don, forme, et raison de l'échange dans les sociétés archaïques. Formulémoslo de otra manera: ¿cuáles son las lógicas que subyacen en la realización del donar?

\section{LA RECIPROCIDAD EN LA TEORÍA DEL DON}

La donación es, fundamentalmente, una forma de intercambio, en la que dos actoras $^{3}$ establecen una comunicación a través de un medio. Cualquiera que sea su forma, toda transmisión, al poner en relación actantes a través de lo dado, implica una relación, y, aunque no sea lingüística, sí es paralingüística. Las comunicaciones humanas precisan algún tipo de norma que regule las condiciones y el marco contingente en el que se produce la comunicación. Si no se comparte ese metalenguaje, la comunicación, y por extensión cualquier intercambio, es imposible. Los metalenguajes se plasman en diversas instituciones y dispositivos ${ }^{4}$

\footnotetext{
${ }^{2}$ Lógica: estructura de una dinámica, como articulación endógena de un conjunto de prácticas.

${ }^{3}$ Actora: cualquier actante autopoyética; actante: cualquier entidad que actúa en un sistema social.

${ }^{4}$ Un dispositivo lo definimos como un proceso en el que fin y medio son resultado de la situación de la actante en el mundo, lo que marca su interacción en el medio (hacia el medio, hacia ella misma y hacia otras actantes). El dispositivo carece de intencionalidad, ya que no es ni posterior ni anterior a la constitución de la actante, sino que su funcionamiento determina a la actante. No es simplemente instrumental hacia la obtención de un resultado, ya que, tanto el resultado de la acción, como lo que la lleva a cabo, son consecuencia del dispositivo; de ahí que se hable de activación o emergencia.
} 
que regulan el intercambio de forma explícita, como contrato, o implícita, como consenso o ambas, manteniendo una misma lógica. Según el tipo de intercambio encontramos uno $u$ otro metalenguaje que es a la vez medio y contexto: en el lenguaje hay la misma convención de significados y correspondencia simbólica; en el comercio, el mismo sistema de conversión del valor de un objeto en otro (trueque) o en un valor estándar (moneda); en el matrimonio, se intercambian personas entre comunidades o grupos de acuerdo a los sistemas de parentesco. Ya Claude Lévi-Strauss se ocupó de destacar la importancia del intercambio con su explicación de la prohibición del incesto, como mecanismo que garantiza el establecimiento de alianzas, regulando, de esta manera, la endogamia por medio del intercambio de mujeres. Es a la luz de esta nueva interpretación cuando LéviStrauss $(1983$; 1991: 32-42) propone el intercambio como principio básico de la constitución de lo social.

Con el don se activa una forma de intercambio que responde a un metalenguaje que hay que desentrañar. ¿Acaso constituye la donación de sangre una forma de don? Sí y no; afirmo y niego a la vez sin ningún rubor. Sí, porque la donación -en un marco voluntario, no lucrativo y universal- es un regalo, y, en consecuencia, se sitúa fuera de la lógica del intercambio mercantil. No, porque la forma en que se efectúa huye de las características del don, y de ahí deviene parte de su peligro: la indeterminación de las bases que rigen el intercambio, quedando éstas, en muchas ocasiones, ocultas para las personas, lo que favorece que se empleen estrategias de acción que entran en conflicto con los objetivos buscados desde la biomedicina.

En tal caso, parece que la donación de sangre es un tipo irregular de don que no se ajusta a sus principios rectores, pero que tampoco está libre de ellos. Esta doble indeterminación (¿qué tipo de don es? ¿qué aspectos del don siguen activos en ella?) no negará la lógica del intercambio.

María Moliner (1998: 1034) define donar como: "Dar a alguien voluntariamente una cosa o el derecho que se tiene sobre ella". Si desglosamos esta definición, donar es:

1. Dar. Un contacto en el que cuatro elementos entran en juego: cada una de las actoras que está en uno de los polos de la relación (donante y receptora), lo que se transmite (sangre) y las condiciones que rigen esa relación (las condiciones de la donación, la consideración de las actoras en cuestión, la práctica biomédica y la legislación actual sobre transfusión); es, en definitiva, una forma de comunicación.

2. A alguien. Las actoras implicadas en cualquier proceso de comunicación son personas, ya jurídicas, morales o individuales. La particularidad de la donación de sangre viene dada por el anonimato y la entrega efectiva a la institución.

3. Voluntariamente. Lo dado no se da con el fin de obtener una restitución, aunque ésta esté implícita. La palabra latina deriva del griego gratia; el adjetivo gratus define la esencia tanto de lo que se da a cambio de nada, como de lo que 
RIS

REVISTA INTERNACIONAL DE SOCIOLOCIA

№ 34, Enero-Abril, 2003

DAVID CASADO NEIRA

se devuelve; el servicio gracioso genera un sentimiento de gratitud, de agradar a quien lo hace y a quien lo recibe (Benveniste, 1983: 129-130). Es un concepto ambivalente que implica una reciprocidad y define las dos partes en cuestión: se da gratis y se recibe gratitud. En la donación de sangre es un marco jurídico el que impone y garantiza la gratitud de la sangre - ni se paga ni se cobra y se da voluntariamente.

4. Una cosa. Con respecto a la sangre, esa cosificación y delimitación es más confusa. La sangre no es un objeto, está despersonalizada —en una transfusión lo que se manipula son bolsas de hemoderivados-; no es una cantidad, aunque es susceptible de ser cuantificable en unidades de sangre extraída - tras el proceso de medicamentalización -, o en valor monetario - con la mercantilización —; y tampoco es un bien, en gran medida, por toda la carga simbólica y emocional que contiene. La sangre, además, no reside en un lugar determinado de la gềografía del cuerpo. En sí misma carece de una corporeidad característica; solamente cuando es derramada es perceptible, fluye por el sistema circulatorio, ocupa el lugar del continente. Incluso para el personal médico resulta difícil responder a la pregunta: ¿dónde está la sangre? La sangre está en las venas y arterias sí, pero también pasa por el corazón y es parte de todos y cada uno de los otros órganos $y$ tejidos del cuerpo humano. La sangre perturba la seguridad de la anatomía (Mol y Law, 1994: 642); somos nuestra sangre en nuestra corporeidad.

5. O el derecho que se tiene sobre ella. En este caso, la sangre se percibe como algo que es propio, que, como parte del cuerpo, no es una propiedad, sino parte del propio ser, del mismo modo que las partes que nos comunican sensorialmente con el exterior:

«Podemos decir que uno "posee" su propio cuerpo o que "le pertenece" (al menos en las extremidades y las partes móviles) en virtud de un flujo constante de información que llega desde fuera, que aflora incesantemente, a través de la vida, los músculos, las articulaciones y los tendones. Uno se tiene a uno mismo; uno es uno mismo, porque el cuerpo se conoce a sí mismo se confirma a sí mismo, en todas las ocasiones, por ese sexto sentido. Yo me preguntaba en qué medida podría haberse evitado el dualismo absurdo de la filosofia a partir de Descartes si se hubiese comprendido de forma adecuada este fenómeno de la "propiocepción"» (Sacks, 1998: 63-64).

Este incierto sentimiento de propiedad personal contrasta con la práctica biomédica, que entiende la sangre como un fluido biológico anónimo, y con las concepciones jurídicas en las que la concibe como parte patrimonializable del individuo ${ }^{5}$.

\footnotetext{
${ }^{5}$ Sobre la cosificación del cuerpo en el derecho anglosajón —en el caso estadounidense- $-\mathrm{y}$ en derecho el romano - en el caso francés-, véase Borrillo (1994); sobre la patrimonialización estatal de la sangre y sobre la regulación del cuerpo como un bien común, véase Lemennicier (1991).
} 
No es solamente arriesgado hablar de propiedad como tal —está la disyuntiva de no reconocernos como propietarias de nuestra sangre, pero sabiendo que es nuestra y parte de nosotras-, sino que, además, sí es algo propio que pasa a ser de otra persona al delegar ese derecho a una tercera entidad - biomédica- que decide a quién y cómo se redistribuye. Lo que se posee circula.

Hay un tipo de relación social fundamental y persistente que queda articulada por un intercambio que reclama una devolución; devolución que no tiene por objeto la obtención de una plusvalía en valor material. Hay intercambios no lucrativos que son hechos por placer, por la constitución o consolidación de un vínculo, pero también por objetivos más inmediatos y palpables. Son todos estos los que ocupan el interés de antropólogas, economistas y filósofas que intentan descubrir la justificación de esta dinámica difícil de encuadrar en la concepción utilitarista de corte capitalista. Bajo ésta, todos los intercambios sólo se conciben paradigmáticamente con un objetivo lucrativo o interesado, bien declarado o bỉen implícito. En este sentido, lo que de momento vamos a tratar como reciprocidad responde a esos ámbitos en los que el intercambio podría estar regulado por los principios utilitaristas, pero que, de hecho, no es así.

Hay otro tipo de intercambio que implica reciprocidad pero, a diferencia del don - dado como la esperanza, la obligación, de la contraprestación-, es regido por medio de un contrato. Sólo en las formas donde se da o se toma algo con el fin directo y único del lucro o de la devolución, hay una norma explícita que lleva al comercio y al préstamo o a la acción razonada. Así se recoge en las teorías clásicas del intercambio, coincidimos con Peter Ekeh (1974) en ese doble enfoque que se polariza en la teoría de la acción racional y en la teoría general del intercambio social. Este segundo referente es el que va a ofrecer una mayor consistencia para poder tratar la donación de sangre en toda la complejidad que adquiera como don, a partir de las nociones de reciprocidad como principio social básico más allá del mero intercambio utilitarista.

Pero es esa concepción utilitarista la que está detrás de las campañas de promoción de la donación, los bancos de sangre echan mano del marketing social para organizar y poder racionalizar las decisiones de las donantes, de esta manera las donantes:

«Son personas sanas que donan su sangre a cambio de una especial sensación de satisfacción. Una sensación de haber hecho algo importante para sí mismo y/o para los demás. El banco de sangre le oferta esa sensación de utilidad y necesidad a través de una donación de sangre segura, agradable y responsable, en la medida en que se le informa de cualquier anomalía analítica y se le orienta ante cualquier hallazgo: anemia, hepatitis, HIV. El banco de sangre le garantiza una cobertura de las necesidades de productos sanguíneos de la población a la que pertenece. Los donantes de sangre obtienen la satisfacción de solidaridad a cambio de tiempo, malestar, miedo y sangre que donan. [...] El marketing requiere que a través de la donación, se satisfaga la autoestima del donante» (Rodríguez Villanueva, 1994: 23-24). 
RIS

REVISTA INTERNACIONAL DE SOCIOLOCIA

No 34, Enero-Abril, 2003

DAVID CASADO NEIRA

¿Por qué la gente quiere satisfacer su autoestima, y manifestar su altruismo y solidaridad? En el caso de encontrar una respuesta convincente, ¿por qué precisamente donando sangre? La explicación de la motivación por altruismo supone una tautología, ya que todas las donantes tienen, por el hecho de donar, un comportamiento altruista. El altruismo no es causa de la donación, sino su consecuencia y manifestación. En un marco de donación voluntaria, no remunerada y universal, toda donación es irremediablemente altruista. A esto hay que añadir la pregunta siguiente: ¿por qué personas que muestran un comportamiento altruista en otros ámbitos no donan, o son decididamente reticentes a donar en otras ocasiones o circunstancias, o, incluso, lo son hacia otras formas de altruismo?

Voy a aventurar una hipótesis que será parte de un desarrollo posterior: tanto la sangre como el tiempo invertido, el malestar o el miedo no se pueden disociar del análisis de la donación, y no se pueden comprender unos, sin comprender los otros. Ambos responden, en una esfera diferente, a una entrega total de la persona, a un comportamiento moral que afecta al cuerpo como una unidad vivencial, y no a una cesión sobre el organismo. Por lo tanto, habría que contemplar la donación como una entrega absoluta de la persona, una entrega que es peligrosa porque implica una pérdida de control y la posibilidad de sufrir una agresión a la identidad personal; y no simplemente como la entrega de un fluido corporal efectuado gracias a que hay un mecanismo de compensación. La acción racional se identifica fácilmente, pero no es el único ni el principal componente de este tipo de intercambios, lo que hace necesario ampliar la perspectiva de análisis.

La transcendencia reside en el compromiso que queda establecido entre las partes. Compromiso que no se limita a una restitución de lo recibido, porque queda fijado un vínculo que será renovado u obviado con las consecuencias que implica cada una de estas decisiones. La importancia del vínculo que se establece o renueva, tampoco queda al margen de las reglas de funcionamiento de las redes sociales que también conforman el don, redes en las que llega a ser fundamental la localización de esa persona a quien va destinada la entrega; a la ventaja de la ayuda anónima, que no implica compromisos, gana el peso de la alianza preexistente.

La persistencia de la clausura o delimitación de los límites de la comunidad —no está de más recordar el paralelismo metafórico que Douglas (1991: 143) establece entre el peligro de transgresión de los límites del cuerpo y las fronteras de la comunidad-, también se presenta en la experiencia de la donación actual. Metafórico, porque hay una continuidad simbólica entre el límite de la comunidad y el del cuerpo, pero que no es expresado en términos de una filiación sanguínea, sino de transgresión de esos límites dados por los lazos del intercambio, bien factible o bien rechazado. El intercambio altera otras fronteras peligrosas y contaminantes; el establecimiento de un lazo invoca ese peligro, el cual se establece en este caso por la sangre, pero que debe su transcendencia a la importancia que tiene para la conservación de la vida, antes que a la delimitación 
de una genealogía sanguínea. El "ordenamiento de la jerarquía social" (Douglas, 1991: 146) reside en la microfisica del control del intercambio, de los lazos y de las alianzas.

El contrato anula el don porque hace obvio el intercambio, se eliminan conjuntamente la restitución, la gratitud y las plusvalías morales generadas - de alianza, confianza, placer y prestigio-, que conforman el don. De hecho, las premisas iniciales de Mauss y de otros muchos autores clásicos del don ya alumbraron otros aspectos de la microfisica del poder en la transfusión y de las dispares relaciones entre lo institucional y la población general, también contribuyeron a fijar el denominado altruismo como una pauta de comportamiento que responde a una coherencia, de acuerdo con la cual es difícil ver una clara línea de división entre éste y el egoísmo. Pero no olvidemos que la racionalidad instrumental no está sujeta necesariamente a los principios de la maximización del beneficio, ya que, además de lo obtenido, entran en juego distintas relaciones de fuerza articulados por la gratitud y las plusvalías morales —en particular el placer, ese grande olvidado en el dar.

\section{¿QUÉ GENERA EL DON?}

Las primeras aproximaciones teóricas sobre el intercambio nos llegan de la mano de la antropología en el ámbito económico, con el estudio de los fenómenos comerciales no lucrativos, que quedaban al margen de la lógica económica de las sociedades capitalistas (Firth, 1974: 9-16). Una de las aproximaciones mejor documentadas desde la antropología clásica a los sistemas de intercambio, la llevó a cabo Bronislaw Malinowski (1973) en el estudio del kula. Cuando Mauss toma el material etnográfico dedicado a estas formas de intercambio, le da una transcendencia que lo saca del constreñimiento de la primera interpretación economicista, y lo eleva a principio básico de reproducción y organización social.

En su obra, Mauss hace una reinterpretación del kula como un metalenguaje de intercambio no lucrativo, poniendo de manifiesto un nuevo significado del regalo. En su ensayo formula que el don - como tal es entendido en situaciones de voluntariedad y prestaciones gratuitas - provoca, en primer lugar, una lógica sujeta a tres obligaciones (dar, recibir y devolver) y, en segundo, que, en los intercambios, las personas individuales, morales, y lo que se intercambia, vienen a ser a misma cosa, como parte de lo que define como "sistema de prestaciones totales" (Mauss, 1991: 160).

Las obligaciones del don. Las tres obligaciones del don (dar, recibir y devolver) provocan una inercia, una dinámica que convierte el acto de dar en un don. Independientemente de las diferentes manifestaciones del don, esta lógica que se desencadena responde a la esencia del intercambio, que tiene como consecuencia el establecimiento de un vínculo de reciprocidad en el que tanto quien da, como 
RIS

REVISTA INTERNACIONAL DE SOCIOLOCIA

№ 34, Enero-Abril, 2003

DAVID CASADO NEIRA

quien recibe, quedan ligados a sucesivos compromisos ${ }^{6}$.

En un primer momento, una persona da a otra, la cual recibe. A su vez, quien recibe, devuelve, y en consecuencia también da. Para que la devolución sea efectiva, ésta tiene que ser aceptada, con lo que quien originariamente dio se transforma en receptora. A partir de ese momento ninguna de las dos actoras puede ya simplemente dar, sino solamente devolver. De esta manera, queda constituida una alianza entre ellas, y así continuamente, a no ser que en un ciclo una de las partes no cumpla - devolviendo lo recibido-, rompiendo la lógica del don y el pacto implícito. Tanto dar como aceptar un don es un acto trascendental, por el vínculo establecido, una alianza, y por su condición, recurrente.

La lógica del don ofrece un circuito que se cierra sobre sí mismo, pero no es contradictorio: necesita unas reglas muy precisas sobre cómo dar, recibir y devolver. En primer lugar, las reglas del intercambio del don permanecen implícitas y, de la misma manera, son aceptadas —el refranero ilustra bien esta lógica: "Dame y te daré". Se impone una correspondencia entre lo dado y lo devuelto, que también hace posible que las dos partes cumplan con su papel. Se da lo que va a poder ser devuelto: "Mi pariente Pedro es bueno; tanto me da él, tanto le doy yo", lo que hace posible que ambas partes cumplan con su compromiso, es decir, la obligación de satisfacer los tres momentos del don: "A quien algo le hayas negado, nada le pidas". Pero el don tiene trampa, los compromisos de la alianza nos sitúan muy lejos del ideal romántico precapitalista en el que nada tiene precio y todo se obtiene a través del intercambio. El don no queda al margen de la racionalidad instrumental, ni del peso de la alianza, porque, en el fondo, el don presenta una completa falta de inocencia, que responde al interés de que antes o después, directa o indirectamente, se espera sean restituidos, con lo que la voluntad aparece como una obligación moral derivada de la aceptación del don; así que, "Muchas veces, lo dado, aunque gratis, es caro". Tanto el interés como la no voluntariedad son lo que le confiere tanto poder de realización, porque, como refleja Mauss (1991: 157):

«[Hay algo] aparentemente libre y gratuito y, sin embargo, obligatorio e interesado de esas prestaciones; prestaciones que han revestido casi siempre la forma de presente, de regalo ofrecido generosamente incluso cuando, en ese gesto que acompaña a la transacción, no hay más que ficción, formalismo y mentira social, y cuando en el fondo lo que hay es la obligación y el interés económico».

\footnotetext{
${ }^{6}$ Autores, como Godelier (1998) o Lévi-Strauss (1991), críticos con la explicación de Mauss sobre la obligación en el don (hau), cuestionan su origen, pero no su existencia.

${ }^{7}$ Todos los refranes fueron extraídos de Ferro (1987).
} 
Pero la satisfacción del interés económico ni es hecha de una manera inmediata, como es el caso del comercio, ni es la principal, porque el objetivo primero es el establecimiento de redes y alianzas, más allá de las simples prestaciones, ya que el don presenta, además, otros componentes. Alfred Radcliffe-Brown encontró un ejemplo muy claro de esas facetas implícitas en el don entre los adamans:

«A pesar de la importancia de estos cambios, como el grupo social y la familia son autosuficientes en cuanto a útiles, etc., estos regalos no tienen la misma finalidad que el comercio o el cambio, en las sociedades más desarrolladas. La finalidad es fundamentalmente moral, el objeto es producir un sentimiento de amistad entre las dos personas en juego, y si no se consigue este efecto, la operación resulta fallida» (Radcliffe-Brown, A. R. 1922, citado por Mauss, 1991: 177).

Alianzas frustradas que son negadas o alianzas que son rotas. "En el tiempo de los higos no hay amigos", pero quien acepta lo dado, también acepta implícitamente la norma del intercambio, conformando asi una comunidad de reciprocidad, una alianza implícita. La devolución es el acto que consolida la alianza cuando es en forma de contraprestación, un contra-don. No se devuelve lo mismo que se recibe, porque lo restituido gana en valor, porque lo que es dado de vuelta es comúnmente algo diferente a lo recibido y, además, es postergado. En otras palabras, el don, para ser tal, demanda una reciprocidad adquirida a través de una obligación moral que queda sellada por el tiempo. La ritualización del don se hace a partir del tiempo: el don se devuelve a posteriori, o simultáneamente, en una ceremonia cíclica. Y tanto la demora en la restitución, como la alianza asumida, hacen obviar la necesidad de un contrato formal. La confianza depositada en la otra parte solamente se certifica aceptando la incertidumbre y a la espera de una contraprestación en el momento oportuno, o en la repetición del intercambio en un nuevo ciclo.

\section{Contraprestación: la asimetría de lo restituido}

Se devuelve lo que se recibe, algo que es lo mismo. En esta lógica, en la que lo dado adquiere un valor moral, más allá del estrictamente utilitario, comúnmente lo devuelto es de diferente condición y entregado con posterioridad. Aunque sea igual, adquiere otra esencia, porque lo restituido gana en valor; además, el don demanda una confianza en la devolución - que ésta sea realizada con algo de valor proporcional- que queda también establecida en la demora, en la postergación de la restitución.

En consecuencia, como devolver implica dar lo mismo de vuelta, vamos a utilizar el término contraprestar (dar lo equivalente de vuelta sujeto a un incremento de valor moral) para dejar patente esta diferencia; tal como indica Gonzalo Abril (1986: 72), "no se trata de restitución, sino de recursos jurídicos o micropolíticas, 
RIS

REVISTA INTERNACIONAL DE SOCIOLOCia

№ 34, Enero-Abril, 2003

DAVID CASADO NEIRA

de la interacción que modifican así sus relaciones tanto como los menudos engranajes del orden social". Contraprestar incluye la restitución sometida a las cuatro plusvalías morales y a un interés que convierten algo en un don. Abordémoslas con detalle:

a) Plusvalía de la alianza. De una manera oculta, con la aceptación del don se entra en una red de reciprocidad; una ya no está sola, ahora tiene la posibilidad de acudir a otras para obtener aquello de lo que no dispone por sí misma. Lo dado es recibido; se constituye como símbolo e implica una alianza. Como se recoge en una de las entrevistas: "Donar sangre vincula porque estás dando algo de ti. O sea, algo que no se compra con dinero" (Informante 1). Tal es así que en esta doble lógica de la obligación recíproca, la no aceptación del ciclo del don tiene consecuencias graves de enemistad o aislamiento en cualquier momento del ciclo del don porque "tanto negarse a dar como olvidarse de invitar o negarse a aceptar, equivale a declarar la guerra, pues es negar la alianza y la comunión" (Mauss 1991: 169-170). Tal como nos recuerda la informante 2 hablando de los lazos vigentes en diferente grado:

«Unos somos muy interesados y otros no somos interesados. $Y$ hay tareas en las que yo participaba mucho y en las que los vecinos se ayudaban todos a todos en algunas cosas: en la matanza, en la trilla, en la siega. En todo eso, todos ayudaban a todos y no te planteabas lo de "yo le ayudo porque me vino a ayudar". No, de toda la vida nos ayudamos unos a otros. Y sin otra cosa, porque también a lo mejor nunca se dio el caso de decir: "Pues este no vino. Entonces, si no me vino a ayudar, yo ahora no le voy a ayudar". Si en algún momento se diera ese caso se rompería con todo».

La estructura de la reciprocidad que implica el don aparece donde ya anteriormente había vínculos sociales establecidos - con lo que se refuerzan con un mayor compromiso recíproco y adquieren una dimensión nueva - o es la invitación para el establecimiento de nuevas relaciones. La alianza absoluta que Mauss ve en los sistemas de prestaciones totales parece limitada a aquellos casos en los que todos los individuos de una comunidad pasan a adquirir el compromiso de la alianza establecida a través del don, en la que todo es obtenido por intercambio. El vínculo establecido está sujeto a una obligación rigurosa:

«En los derechos y economías que nos han precedido, jamás se verá el cambio de bienes, riquezas o productos durante la compra llevada a cabo entre individuos. No son los individuos, sino las colectividades las que se obligan mutuamente, las que cambian y contratan; las personas que están presentes en el contrato son personas morales: clanes, tribus, familias, que se enfrentan y se oponen, ya sea en grupos que se encuentran en el lugar del contrato o representados por medio de sus jefes, 0 


\begin{abstract}
por ambos sistemas. Lo que intercambian no son exclusivamente bienes o riquezas, muebles y inmuebles, cosas útiles económicamente; son sobre todo gentilezas, festines, ritos, servicios militares, mujeres, niños, danzas, ferias en las que el mercado ocupa sólo uno de los momentos, y en las que la circulación de riquezas es sólo uno de los términos de un contrato mucho más general y permanente. Estas prestaciones y contraprestaciones nacen de forma más bien voluntaria por medio de presentes y regalos, aunque, en el fondo, sean rigurosamente obligatorias bajo pena de guerra privada o pública» (Mauss, 1991: 159-160).
\end{abstract}

Todo es susceptible de ser obtenido por intercambio y potencialmente cualquier cosa será dada en contraprestación dependiendo de las prescripciones existentes sobre la equivalencia de valor. En las sociedades occidentales actuales, en las que el intercambio de dones no es el regulador máximo de la organización y funcionamiento social, encontramos también muestras de las hondas implicacionês que afectan a las diferentes esferas y que aparecen presentes en mayor o menor grado en toda forma de intercambio. La existencia de formas de relación social no mercantiles, no solamente se debe a la persistencia del don en determinadas comunidades (por ejemplo, en el mundo campesino), sino que el hecho de que el lazo personal sea sustituido por un intercambio anónimo más característico de las sociedades modernas no se sustrae tampoco de esta lógica.

El pacto no solamente implica a quien lo hace, sino a quien representa; por lo tanto, afecta simultáneamente a la propia estructura del grupo, llevándolo a nuevas relaciones de poder hacia el exterior. Cómo se considere a la persona en relación al grado de implicación que sus actuaciones tengan para el resto de los miembros del grupo social implica, en un mayor o menor grado, a los demás miembros; en otras palabras, el grado de cohesión vigente determina la respuesta e implicaciones para la totalidad de ese colectivo, tanto en comunidades grupales como en comunidades individualistas. Si bien en el primer caso la propia comunidad va a ser la implicada en el intercambio, en el segundo, estamos en un contexto individualizado en el que esta posición está ocupada por el Estado, regulador de las relaciones sociales. La obligación de la restitución se manifiesta como una obligación asumida, que además va a ser demandada implicando a la persona moral en cuestión. Pero como reza el refrán: "Amistad por interés, no dura porque no la es", la alianza no es el único componente del don.

b) Plusvalia de la confianza. Aceptándose el compromiso, lo restituido no es de la misma condición que lo recibido, pero sí ha de ser equivalente, si no en valor material, sí en valor moral; el sacrificio hecho ha de ser correspondido, simbolizado en lo dado de vuelta. No hay una devolución inmediata, sino que la confianza se articula en la esperanza de la restitución en el futuro, donde lo fundamental es no olvidar los términos ni la alianza. La ritualización del don se hace a partir del tiempo: el don se devuelve a posteriori, o simultáneamente, en 
RIS

REVISTA INTERNACIONAL DE SOCIOLOCIA

N 34, Enero-Abril, 2003

DAVID CASADO NEIRA

una ceremonia cíclica. Y tanto la demora, como la alianza asumida, obvian el contrato formal. El informante 3 destaca sobre esta necesidad: "Hay bancos de sangre donde ahí está para cuando sea necesaria. Y por eso la aporto sin darle más importancia, ni más trascendencia, ni nada. Se necesita sangre, a mi me sobra, pues ahí está". La confianza depositada en la otra parte solamente se certifica aceptando la incertidumbre a la espera de una contraprestación en el momento oportuno, o en la repetición del intercambio en un nuevo ciclo (que constituye otra forma de confianza temporal). Pero, sobre todo, el fin es fundamental para justificar o no el don:

«i¿Tú que sabes que hacen con la sangre después de meterla en la bolsa?! Algunas veces mentirán, porque no sé, habría que ver el porcentaje de accidentes y el porcentaje de necesidad de sangre. Sabemos que siempre necesitan, pero igual también hacen comercio con la sangre. No lo sé, yo eso no lo sé, habría que estar dentro de ese mundo. De la sangre y del sudor se beneficia mucha gente» (Informante 4).

La confianza en la intencionalidad de la otra está presente, siempre que no esté movida por el interés de la obtención de lucro a costa del sacrificio propio, lo que se desvelaría asimismo con el paso del tiempo. Pero, además, hay que aceptar el papel mediador del banco de sangre, de lo contrario se convierte en un agresor identitario: "Dentro de mi cuerpo ya gobierno yo, nadie lo va a gobernar por mí. Eso está clarísimo" (Informante 5). La asociación frecuente entre banco de sangre y vampirismo, bajo la popular imagen de Drácula, no viene únicamente determinada porque el objeto de la apropiación sea la sangre; la idea de una apropiación indebida, interesada y sibilina responde a esa incertidumbre de los fines que rigen la actuación biomédica. Sin confianza no hay don, pero el don genera confianza, como reconoce el informante 6 , donante habitual:

«No tengo temor. Está claro que seguro que en el mundo, que en algún sitio, en algún momento, se comercia con sangre, se comercia con órganos, se comercia con todo eso. Pero en la sociedad que me tocó vivir y en el entorno en el que vivo, creo que no hay ningún tipo de problema con eso».

No es casual que las reticencias contra la donación se reduzcan entre aquellas personas que donan con cierta frecuencia, tanto en lo referido al conocimiento de los aspectos relacionados con la organización, gestión y desarrollo de la actividad transfusional, como en los psicosomáticos (intensidad del dolor, molestia...) (Edwards y Zeichner, 1985; Piliavin y Callero, 2000).

c) Plusvalia del prestigio. La generosidad es la expresión del prestigio; quien $\mathrm{da}$, entra en alianza y quien da de vuelta más, consigue renombre; otras quieren aliarse con ella, y disponen de más posibilidades de establecer y de consolidar 
vínculos de reciprocidad, a la vez que incrementan sus posibilidades de recibir más. Tal es la lógica del potlatch, donde pensamos que muchas de las actuales celebraciones de carácter gastronómico (por ejemplo, un banquete de boda) tienen una finalidad parecida, y como documenta Émile Benveniste (1983: 52):

«Encontramos en indoeuropeo una manifestación social que en el lenguaje de los etnógrafos se denomina potlatch: exhibición y destrucción de riquezas con ocasión de una fiesta. Hay que mostrarse pródigo con los bienes de uno para hacer ver que no se les da importancia, para así humillar a los rivales mediante el despilfarro instantáneo de riquezas acumuladas. Un hombre conquista y mantiene su rango si aventaja a sus rivales en este gasto desenfrenado. El potlatch es una provocación para que los demás gasten a su vez; los competidores hacen un gasto superior, de ahí un circuito de riquezas acumuladas y repartidas para prestigio de unos y goce de los otros».

A la inversa, una persona avara entra en el intercambio, pero no se preocupa de aumentar la plusvalía del prestigio a través del incremento en el valor de lo dado; si alguien generoso es la representación pura de la contraprestación, alguien avaro representa la pura restitución, ya que lo que da de vuelta no está revalorizado. $\mathrm{La}$ pérdida u obtención de prestigio tiene como consecuencia directa una redefinición de las relaciones de poder dentro de la red de solidaridad, colocando a las actoras en posiciones más o menos ventajosas a la hora de cumplir con las obligaciones del don, porque el valor de lo dado revierte en quien da y es un reconocimiento a quien lo hace, en una ambivalencia entre demostración y reconocimiento de poder, ya que el prestigio sólo tiene valor como res pública. Pero el anonimato de la donación de sangre impide que se pueda activar el prestigio en su totalidad, aunque nos encontramos con bancos de sangre como el Centro de Transfusión de Galicia (CTG), entre otros, que se ocupan, a través de notas de prensa o ceremonias anuales, de premiar, reconocer y agradecer públicamente la participación de determinadas personas o colectivos en las campañas de donación. De la misma manera, la informante 7, quien no donó nunca, pone de manifiesto que "la gente siente un orgullo, siente que hizo algo bueno, incluso si a lo mejor su sangre no vale, pero eso no se piensa, sólo se piensa que fuiste a donar y estuvo bien"; informalmente, la plusvalía del prestigio circula dentro de los propios grupos sociales de referencia como marcador de valía.

d) Plusvalia del placer. La satisfacción de dar, recibir y devolver, olvidada con demasiada frecuencia en el tratamiento de los intercambios, no debe ser pasada por alto y es la que contrasta más con la lógica utilitarista. Incluso, ante la variedad de las formas de intercambio de dones, existen casos en los que la satisfacción económica aparece como totalmente relegada a un segundo plano o incluso ignorada - por lo menos si consideramos lo económico en un sentido restringido a lo 
RIS

REVISTA INTERNACIONAL DE SOCIOLOCIA

№34, Enero-Abril, 2003

DAVID CASADO NEIRA

mercantil o al intercambio de objetos- Conocemos en la experiencia cotidiana el placer de regalar y de ser agasajados.

El placer de dar por dar ocupa aquí un lugar central. El carácter festivo, ceremonial, o ambos, que acompaña a la entrega y recepción de un don es una parte consustancial a él, como muestra de la voluntariedad y la satisfacción de dar. Éste no está ausente tampoco de la donación de sangre, lo que se manifiesta, por ejemplo, en la alegría de dar. Como indica la informante 7: "Hay gente que siempre quiso donar, o siempre tuvo ilusión por donar sangre, que la hay. Yo conozco gente que tan pronto ven venir al autobús [unidad móvil] se alegran un montón, por donar"; o la informante 1, quien participa frecuentemente en las campañas de donación: "Sí, pero en mi caso particular a mí me gusta donar sangre, porque sé que hace falta, y a mí me gusta. Me siento bien haciéndolo y me siento mejor que haciendo otras cosas". El placer de dar surge como una satisfacción personal en esa proyección hacia otras personas, una satisfacción que hace destacar la limitación del egoísmo/altruismo como una contradicción irreconciliable, no exenta de los compromisos del don.

e) Gratitud. Hay que considerar otro elemento, que sin ser plusvalía también es indisoluble de la contraprestación. Parte de la alianza, la gratitud es un reconocimiento a la otra actora, que, en el juego del equilibrio inestable del intercambio, opera como un estabilizador de la simetría recíproca, como un catalizador de la obligación de restituir: "El agradecimiento es un nuevo merecimiento", la justificación moral del devolver. Ésta constituye la parte pública de la obligación contraída. Cuando solamente hay obligación e interés, nos encontramos ante otras formas de intercambio ni gratuito ni voluntario, como el comercio o el estupro, que están hechas de acuerdo a un contrato, a un patrón de valor (moneda) o a una esclavitud por violencia directa. Pero también existen formas de intercambio gratuito y voluntario pero establecidas con la intención de obtener un beneficio sin contraprestación, o mayor del que se espera restituir (en valor o en gratitud); se trata del oportunismo, que atenta contra los principios de la reciprocidad: "Quien no agradece, al demonio se parece". El agradecimiento es el reconocimiento explícito (lingüístico o paralingüístico) del compromiso que se genera en la aceptación del don y un equilibrador inmediato de la deuda adquirida. Además de las ceremonias y notas anteriormente mencionadas, nos encontramos con que el CTG en muchas de sus comunicaciones con sus donantes incluye mensajes que cumplen esta función, como por ejemplo, "el Centro de Transfusión de Galicia desea agradecerle su inestimable ayuda al realizar esta donación de sangre"; "gracias por participar en el programa de donación de sangre de Galicia"; o "con su colaboración muchos enfermos pudieron superar su problema de salud e incluso algunos salvaron su vida, gracias a la sangre que usted donó altruistamente". A esto hay que añadir el envío a la donante después de cada extracción de una carta de agradecimiento con los resultados de la analítica, en un retorno valorado muy 
positivamente por las donantes, que, además, reclaman que se les devuelva la información obtenida.

En cada forma de contraprestación aparecen necesariamente los cinco elementos - las correspondientes plusvalías y la gratitud que acompañan a lo restituido-; es la intensidad de cada uno de ellos lo que diferencia una u otra forma de don, pero, necesariamente, están presentes todos ellos en mayor o menor medida. El conjunto se mantiene estable aunque cambie la intensidad de sus componentes; un incremento en el valor de lo restituido, en la gratitud o en alguna de las cuatro plusvalías, genera igualmente un aumento en todas las magnitudes -en la misma medida o en diferente proporción. La plusvalía del prestigio se basa en un incremento del valor de lo dado (valor moral o material según se trate de sacrificio o de gasto); la del placer, en el gozo lúdico y la satisfacción; la de la confianza, en la asunción del riesgo de la restitución; la de la alianza, en el beneficio recíproco; y la gratitud, en la consideración de recibir más de lo dado y en la obligación moral de la contraprestación.

Lo dado en el primer momento del ciclo del don, en la propuesta de alianza, solamente transmite lo que es dado, más las cuatro plusvalías. Solamente cuando se acepta lo dado, se realiza como don, al entrar el elemento gratitud (de recibir y de que se acepte lo dado) en juego. De esta manera queda sellada la promesa implícita de devolución. Una vez devuelto pasa ya a ser don porque es contraprestado.

La asimetría (en el tiempo y en lo restituido) que caracteriza a la contraprestación es precisamente lo que le confiere poder. Contrariamente a lo que parece a primera vista, en este vacío provisional y recurrente (entre quien da y recibe sucesivamente) radica la simetría y el equilibrio de la relación, porque lo que se constituye es el vínculo de reciprocidad, imposible en una simple devolución. Ésta, lejos de ser su negación, es la esencia del intercambio. Raymond Firth, en una crítica desde la antropología funcionalista a la idea de obligación de devolución de Mauss, pone como ejemplo su experiencia personal durante el trabajo de campo:

«Al igual que otros antropólogos, mi esposa y yo, de regreso en Londres, no nos sorprendimos de recibir algunas solicitudes de dinero por parte de malasios que habían sido nuestros informantes y amigos en Kelatan. En frases amables, las cartas (originalmente escritas en malasio y por un pariente o amigo) solicitaban ayuda financiera por los gastos de la boda de una hija, la caída de una casa, la capilla del poblado que iba a ser reparada o una estación de pesca muy pobre. Como muestra transcribo parte de dicha carta traducida. "M envía esta carta. Porque $M$ desea pedir ayuda a Tuan. Porque $M$ en este momento se encuentra en grandes dificultades. Porque el poblado de $M$ ha sufrido un gran monzón y la lluvia ha sido excesiva. $\mathrm{N}$ [hijo de ella] no ha podido salir a pescar porque el mar ha estado muy picado. Por ello, M solicita ayuda en dinero; si Tuan y Mem se compadecen de $M$, envían dinero en la medida de los deseos de Tuan. $\mathrm{N}$ se acuerda mucho de Tuan y Mem porque Tuan y Mem fueron de corazón gene- 
RIS

REVISTA INTERNACIONAL DE SOCIOLOCIA

№ 34, Enero-Abril, 2003

DAVID CASADO NEIRA

roso y muy buenos con él. $\mathrm{N}$ considera a Tuan como a su padre y a Mem como a su madre. $M$ recuerda también el tiempo en que Tuan y Mem vivieron en su casa y toda la comida que ella preparaba le gustaba a Tuan. Si Tuan quiere, M puede enviarle algo de dulce de coco..."» (Firth, 1974: 20-21).

La petición de dinero por parte de las antiguas informantes y anfitrionas se ajusta perfectamente a la lógica del don, ante la que Firth es ciego. Él se niega a la ayuda porque ya no está en el lugar y no ve comprometidas sus relaciones ("habian sido nuestros informantes y amigos"). En su situación de retornado no sigue inmerso en las redes de relaciones interpersonales de las que se puede sustraer. Cabe pensar que, estando en el lugar, su negativa tendría consecuencias (enfado y decepción en la familia de M; dificultades de integración; pérdida de privilegios para Tuan y Mem). Aquí están en juego, tal como se refleja en la carta, la alianza, la gratitud, el prestigio, el placer y la confianza. Esta demanda se fundamenta en la existencia de un vínculo anterior basado en el gozo de anteriores dones materiales e inmateriales ("M recuerda también el tiempo en que Tuan y Mem vivieron en su casa y toda la comida que ella preparaba le gustaba a Tuan") y en la existencia de alianza y prestigio ("N considera a Tuan como a su padre y a Mem como a su madre"), por lo que no se pide una devolución, ni un pago ("envían dinero en la medida de los deseos de Tuan") sino contraprestación, lo que permite ofrecer "algo de dulce de coco" a cambio de "ayuda en dinero". Con su negativa, Firth lleva a cabo la ruptura de una vieja alianza; ya no le interesa o se le escapa la lógica y consecuencias del don. Julio Caro Baroja plasma esa disyuntiva: "El don, pues, desde el punto de vista sobrenatural o prenatural, es algo que queda fuera de las ideas de justicia, reciprocidad, etc. [...] Pero en la vida común y corriente cada paso, cada relación contraída, se expresa por un don o, menos solemnemente, por un regalo. El regalo es un vínculo" (Caro, 1986: 8).

Firth confunde la lógica del don con la del otro tipo de intercambio por excelencia, el comercio, pero éste, a diferencia del don, es regido por medio de un contrato. Sólo en las formas en donde se da (o se toma) algo con el fin directo y único del lucro en la devolución, hay una norma explícita que lleva al comercio $\mathrm{y}$ al préstamo. El contrato anula el don porque hace obvio el intercambio; elimina la plusvalía, la restitución y la gratitud que conforman el don:

«Así, el regalo no es obviamente una versión hipócrita del préstamo, sino un intercambio que se basa en otro tipo de estructura antropológica. El régimen del préstamo es el contrato, a saber, un acuerdo explícito de contraprestación. El régimen del regalo es el consenso, un acuerdo implícito y basado en la confianza. El silencio consensual sobre la obligación de restituir no responde inicialmente a los términos de "ideología" neolítica o burguesa alguna. Responde a la naturaleza misma del lazo simbólico, que requiere de un tercer término secreto (el excedente, lo sagrado), mediador de la reciprocidad» (Abril, 1986: 68). 
Opuesto a la contraprestación, en el contrato se garantiza la simetría de lo devuelto y se determina un período (inmediatamente o pospuesto a fecha fijada) sin recurrir a la confianza. La alianza es un simple acuerdo en el que el componente moral (gratitud y plusvalías) es insignificante. La mercancía o lo prestado circulan bajo la norma contractual, perdiendo así todo el carácter simbólico (confirmador del vínculo de reciprocidad) que es el elemento sagrado del don, el hau. Así queda también expresado en la cultura oral: "El que debe y paga, no debe nada". El pago anula todo deber (excepto el propio pago) evitando, como se recoge en este refrán, cualquier vínculo de compromiso transcendente entre las actoras envueltas en el intercambio, porque los compromisos implícitos son mayores que mediando pago: "La dádiva es más cara que si fuera comprada".

\section{LA SANGRE COMO DON}

La voluntariedad en los intercambios está condicionada, en primer lugar, por la obligación del dar, recibir, devolver, que constituye una forma de compromiso en el que se reconoce el vínculo que se establece a través de lo dado, y en el que hay un juego de equilibrio entre las partes. Pero el don no es inocente, ni tampoco las relaciones sociales, por eso, hay que tener en cuenta que hablar de simetría entre las partes es ilusorio, pero también eso es lo que le otorga fuerza al don, que ejerce de canal mediador en la lógica de las relaciones de poder. En la dialéctica del poder el grado de negociación factible entre las actoras se presenta como línea directamente relacionada con el grado de voluntariedad. Tanto por el grado de voluntariedad, de simetría, o por la condición de lo dado, hay formas de devolución que son formas de contraprestación totales, otras, que solamente satisfacen dos factores de la contraprestación (gratitud y plusvalías), y otras que son restituciones. En algunos casos, es la condición de la actante dadora o receptora lo que determina una forma u otra de intercambio, desde el don a la donación de sangre.

Ante las diferentes formas de intercambio, la donación de sangre supone ya una forma rara de intercambio que la sitúa en un terreno complejo, donde la ausencia de consenso y la circunstancialidad del contrato hace difusas las reglas y la micropolítica del don.

Cuando lo dado es un excedente y no se busca la obtención de beneficio cambia el tipo de intercambio. La limosna y la donación están destinadas de quien que tiene un excedente (como la sangre, que se regenera) a quien no tiene lo que determina una asimetría de posición entre las dos actoras en cuestión. El sacrificio se limita a la toma de decisión de dar algo y no se produce ninguna de las plusvalías fundamentales para establecer una alianza. El momento de la devolución (muestra de gratitud) solamente es inmediato; no hay posibilidad de postergación que permita algún tipo de vínculo. La plusvalía de la alianza y de 
RIS

REVISTA INTERNACIONAL DE SOCIOLOCIA

№ 34, Enero-Abril, 2003

DAVID CASADO NEIRA

la confianza, ya que no se da aguardando una contraprestación, no se producen; solamente la gratitud, la plusvalía del placer y del prestigio son posibles por el acto de la voluntariedad de dar, como se evidencia en la limosna.

Marcial Gondar (1993: 77-113) explica la reticencia de dar limosna fuera del atrio de la iglesia de acuerdo con la concepción de la fortuna y del trabajo; la limosnera solamente es aceptada cuando tiene la capacidad de poner en contacto lo humano con lo divino. Se evita establecer cualquier inicio de alianza con la pobre en la vida diaria, pero, en el momento de comunión con Dios, la pobre es a la vez una ocasión para demostrar nuestra virtud y es nuestra mediadora ante Dios, como vehículo de ofrenda. La limosna, correspondiendo a un intercambio intrahumano, tiene la particularidad de actuar como mediadora con lo humano, característica de la que carece la donación. La necesidad que justifica la petición es diferente en el caso de la limosna y en el de la donación, debido a la distinta jerarquía entre quien da y recibe en uno y en otro caso, a causa de esa demanda (responsabilidad o fatalidad) o el peligro de contaminación nacido de la marginalidad social. En el caso de la donación de sangre, cualquier consideración moral sobre la oportunidad de dar o no es innecesaria; en este caso, la propia demanda vital y la necesidad sanitaria legitiman ya la petición, como nos señala el informante 6, frente al fin incierto de la limosna donde se cuestiona "si está bien darle o no está, o si ese pobre en qué lo va emplear o no lo va emplear", la donación de sangre no presenta doblez: "mi sangre sirve para mejorar el estado de salud de otra persona".

En la donación, el excedente se redistribuye por esa idea de justicia moral; pero no se debe confundir la donación con la limosna, ya que la asimetría en la jerarquización social es irrelevante en la posición que ocupa en la demanda (al contrario que en la limosna), aunque se trate de redistribución de la fortuna, tal como rezaba un eslogan del CTG: "Comparte tu salud. Dona sangre". En la donación de sangre la persona no es responsable del déficit que causa la petición y justifica el dar de diferente modo que en la limosna, atendiendo a la demanda de salud, hasta el momento incuestionable moralmente.

De este modo sólo se produce una contraprestación parcial en la que no hay restitución; únicamente otra actante (el sistema biomédico) puede volver a hacer funcionar el principio de justicia social, haciendo que en un futuro reciba tanto quien en su momento dio, como quien no, desarticulando el principio del compromiso de la contraprestación. Se da por una obligación moral y por el concepto de fortuna; dar es obligatorio como una forma de justicia que equilibra con aquella que posee en exceso. Si bien en la decisión de donar altruistamente está presente esta justificación, en el caso de la donación (ya no posible) a un familiar o alguna persona determinada, hay que devolver el favor de acuerdo a los principios del don; porque aquí reside el mayor conflicto, la persistencia de la lógica del don en este concepto de la reciprocidad (incompatible con la donación de sangre en el nuevo marco de la transfusión). En un tono esperanzado Mauss (1991: 246) nos recuerda que: 
«Felizmente no está todavía todo clasificado en términos de compra y venta. Las cosas tienen todavía un valor venal, si hubiera valores que se pudieran clasificar sólo como de este tipo. Tenemos otras morales además de las del mercader; todavía hay gentes y clases que se conservan en las costumbres de otros tiempos, costumbres a las que nos sometemos, al menos en algunas épocas o en algunas ocasiones del año».

La actualidad del don sigue presente en múltiples esferas de lo social contemporáneo, quizás en manifestaciones menos totales y ritualizadas que las del kula o lo potlach, pero de hondas implicaciones. La difícil pervivencia de formas de don en el seno de las sociedades capitalistas responde a una doble circunstancia: Si bien sigue habiendo formas de don, éstas parecen existir únicamente ligadas al regalo como forma de agasajo, que se manifiesta solamente en la búsqueda del placer y del prestigio - ¿del consumo? Es en este sentido en donde mi apuesta está ligada a la vigencia del don en su componente metalingüístico, en su presencia como principio de ordenación social, aunque no sea tan visible como en los ejemplos que ofrece la antropología clásica. Como se desprende de las dificultades de realización del don como sistema de prestaciones totales, hoy el don ocupa otro lugar, que genera conflictos allí donde la microfisica del poder es conflictiva. ¿En qué punto de la donación de sangre se genera este conflicto?

Con respecto a la donación nos encontramos con una confluencia de los dos aspectos anteriores: la pervivencia de la circulación del don como establecedor de alianzas, y la fractura en la confianza en las instituciones como reguladoras de la circulación de dones, en definitiva, como garantes de la cohesión social.

La donación de sangre no es una donación pura, sino un tipo de intercambio con implicaciones en la reciprocidad, asimetría entre actoras, que podemos analizar como una forma de intercambio compleja bajo la teoría del don. La donación de sangre presenta una oscilación que no permite ajustarla a ninguna de las formas convencionales de don, reuniendo características propias, pero que tampoco se sustrae a ellas. Por ejemplo, aunque considero que habría que ser muy precavidas con estos datos excesivamente genéricos, no deja de ser significativo que una de cada diez ciudadanas de la Unión Europea aceptaría sangre de cualquier persona si supiese que la iba a necesitar en un futuro cercano, pero un cuarto solamente aceptaría sangre de alguien a quien se la hubiese dado anteriormente (INRAEurope, 1995: xii).

El complejo carácter de la donación de sangre como don se desvela cuando Richard Titmuss publica The gift relationship: from human blood to social policy, una obra que se convertirá en un clásico innovador. Con ella el autor sitúa la decisión de donar en un plano diferente al de una simple decisión individual: el don crea un compromiso entre actantes. Se inicia así un camino en la investigación de la donación de sangre que rompe con los enfoques psicologistas centrados en la identificación de las características personales de las donantes. Se puede decir 
RIS

REVISTA INTERNACIONAL DE SOCIOLOCIA

No 34, Enero-Abril, 2003

DAVID CASADO NEIRA

que, sin duda, este autor es el primer teórico social de la donación de sangre. Aquí retomaremos su propuesta abarcando otras dimensiones y redefiniendo la idea matriz de donación de sangre como don. No sin motivo ya Titmuss ha apuntado que la donación de sangre - voluntaria y no remunerada - implica una especificidad como don. La donación de sangre es impersonal en la petición y en el destino; implica cierto grado de entrega personal; la exclusión de dar es por criterios racionales (biomédicos) ajenos a quien da y no culturales; no hay ningún tipo de sanción por negarse a dar; la receptora no tiene obligación de contraprestar, pero, en el caso de querer, tampoco puede agradecer la sangre a la abastecedora originaria; una intermediaria (el banco de sangre) determina si el don es válido (si cumple los requisitos de calidad transfusional) y también el intercambio y el destino. A éstas hay que añadir otros aspectos que sitúan con más precisión la donación de sangre con respecto al don. Aparecen tres irregularidades que hacen problemática la idea de una donación de sangre como donación (genérica) y siquiera como don puro; las irregularidades del don de la sangre ofrecen más luz sobre los otros porqués.

La aportación de Titmuss hay que encuadrarla en el sistema de gestión norteamericano, en el que hasta 1975, la forma predominante de obtención de sangre y hemoderivados era a través de la donación remunerada. Posteriormente, el National Blood Policy y muchas de las agencias de donación deciden instaurar un sistema de voluntariado y no remuneración, que, según indica Jane Piliavin (1990: 444), se debió, en gran medida, a las repercusiones de la obra de Titmuss, en la que criticaba fuertemente el sistema norteamericano basado en la remuneración. A partir de entonces, se compagina el sistema remunerado de las entidades privadas (entre otras, empresas farmacéuticas) con otro no lucrativo, de las entidades públicas o semipúblicas, orientadas específicamente a la transfusión.

Pero más allá de los beneficios de un sistema remunerado o no, estamos ante el debate sobre las bases que han de regir la constitución de una sociedad. Sigue abierta la controversia sobre cuál de los dos sistemas (remunerado o no) ofrece más garantías, habiendo muchos y diferentes análisis que están también muy presentes en el caso de la donación de órganos. En cualquier caso, hay que tener en cuenta cuatro circunstancias: (i) Esta disyuntiva parece tratarse en muchos casos de un enfrentamiento sobre la concepción de lo social que responde a trayectorias históricas en competencia (Europa frente a EEUU), más que a diferencias en la calidad del la sangre obtenida. (ii) Tampoco hay que olvidar el peso que entidades farmacéuticas y biomédicas tienen en nivel estatal en dos de los países con mayor peso en el ámbito de la transfusión (el Institut Pasteur en Francia y la Cruz Roja con sede en Suiza). (iii) Después de los escándalos de contagio de hemofilicos con elaborados de plasma contaminado, que afectó sobremanera a países como Francia o España, la propia industria farmacéutica tiene mucho interés en asegurar la calidad de la materia prima de sus productos terapéuticos. (iv) Este debate no se cierra tampoco con la opción de un sistema no remunerado, ya que, también en 
este caso, la sangre no queda libre de entrar en un flujo comercial, donde la bolsa de sangre tiene un precio o sirve de base para la elaboración de medicamentos con objetivos comerciales.

Titmuss polarizó ambos sistemas como opuestos, abogando por un sistema que se interpreta sin fisuras, pero el don y el altruismo presentan recovecos y contradicciones que lo alejan de ser un sistema ideal de cohesión social. Las consecuencias y condiciones que implica un sistema voluntario con un alto grado de enajenación individual, producto de la tensión con el servicio de transfusión (demandas de disponibilidad, agresión a la identidad y a la privacidad, control y supervisión biomédica, demanda de compromiso...) se obvian en su crítica.

En primer lugar, habrá que desentrañar en qué consiste este uso del altruismo. Para eso hay que tener en cuenta cómo se constituye en cada sistema social el dispositivo de la reciprocidad y a qué lógica social responde. En segundo lugar, el altruismo está presente en la donación, pero por sí mismo no explica por qué se manifiesta a través de la donación de sangre; para eso hay que tomar en consideración otros aspectos intervinientes como la asimilación y confusión entre donación, extracción y transfusión, o los que se activan alrededor de la sangre y del cuerpo.

Dentro del proceso de transfusión, la relación que se establece entre el CTG y la donante es el eje central. En esta relación la donante es ante todo considerada una cliente, alguien que vende un producto (sangre), compra otro (satisfacción del altruismo) y paga un precio (molestias de la extracción); por lo menos, así es como se concibe bajo el marketing social. Pero fuera de este principio organizador, la relación es diferente: En primer lugar, la justificación clientelar no explica por qué se dona; en segundo lugar, la preocupación por la donante implica que lo que el CTG ofrece es una contrapartida y no un pago. El CTG despliega una serie de instrumentos para convertir la relación voluntaria de la donación en una obligación y en un compromiso de fidelización hacia el CTG. En esta concepción, tener la sangre útil va asociado directamente al altruismo, donde moral personal e interés biomédico están confundidos (fundida una con el otro y confusos). Está sana la persona que es extractible. Es en este contexto en el que hay que entender el voluntariado con respecto a la donación. Ya en la primera experiencia británica con donantes voluntarios a partir de 1921, Percy Lane Oliver, con su Servicio de Transfusión de Sangre de la Cruz Roja en el Gran Londres, pone de manifiesto las limitaciones y posibilidades del altruismo en la donación de sangre (Starr 2000: 81-89). Aparentemente, el voluntariado es la forma perfectamente ajustable a la donación, pero sólo lo es parcialmente. Los bancos de sangre están interesados en voluntarios (así lo recomienda la Organización Mundial de la Salud y lo exige la legislación española vigente) pero la lógica biomédica se sustenta sobre la base de que todo el mundo es donante potencial, y se presupone, no se concibe de otro modo, que toda persona sana tiene el compromiso de donar.

Tanto legal como biomedicámente, la sangre es conceptualizada sobre una base patrimonializadora. En una definición estrictamente biomédica, la sangre es "un 
RIS

REVISTA INTERNACIONAL DE SOCIOLOCIA

№ 34, Enero-Abril, 2003

DAVID CASADO NEIRA

tejido líquido circulante" (Bernard, 1998: 13) o, tal como queda recogido para el Consejo de Europa: "Para los propósitos de este Acuerdo, la expresión 'sustancias terapéuticas de origen humano' hace referencia a la sangre humana y a sus

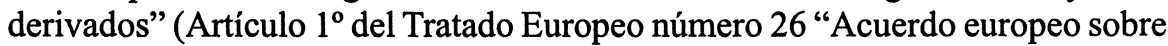
el intercambio de sustancias terapéuticas de origen humano. París 15.12.1958"). Según esto la sangre se convierte en un componente del organismo, o en un producto terapéutico, de la misma manera que el cuerpo pasa a ser la fábrica que lo elabora. Asumir esto supone participar de una concepción de lo corporal biomedicalizada - o civilizada, según Turner (1992) - . La sangre tiene capacidad curativa, pero sólo cuando se somete a esta transubstancialización. Este proceso es una manipulación química (ya no es un fluido vital, es un producto terapéutico) y simbólica (deja de circular por el cuerpo para circular como bien o mercancía). Esta fractura a la que se somete la sangre anula cualquier huella que hubiese de vínculo entre la persona y su cuerpo. Se niega la propiedad de la sangre, a la vez que se trastoca un acto personal en un proceso despersonalizado, en el que la donante sólo tiene valor dentro de la taxonomía biológica de la especie humana, para la que abastece de una sangre en circulación universal. La sangre es de quien lo necesita, y quien establece esa necesidad es la asistencia sanitaria, porque sin ella no hay donación, extracción, ni transfusión. Del conflicto entre la libre circulación (universal) o la circulación sólo restringida surge la diferente concepción de los límites de la comunidad de las actoras, como pasaremos a ver posteriormente.

La forma en la que se efectúa la donación hace que sea un acto mediatizado en el que se establecen demandas muy estrictas y concretas sobre la calidad sanguínea. Quien dona es sometido a un proceso de vigilancia y control exhaustivo e individualizante. La institución biomédica necesita individualizar de acuerdo a los principios que rigen la relación médico-paciente. La existencia de un cuerpo especializado y limitado de donantes (característico de las antiguas hermandades de donantes) contradice la demanda y el compromiso generalizado que se desprende de la universalidad de la sangre y la entrega determinada no por la voluntad individual, sino por la calidad sanguínea y las necesidades sanitarias.

La relación entre quien da y quien recibe no es directa y, por lo tanto, está fuera del control de la donante y de la receptora. La institución encargada de las transfusiones actúa como intermediaria absoluta: ella es la demandante, la receptora primaria, y la redistribuidora a su vez de los hemoderivados producidos; además, ofrece algún tipo de compensación (en forma de tentempié posdonación, envío de resultados de la analítica a casa de la donante, actos de reconocimiento de donantes, etc.). Ahí es incuestionable su mediación debido a la complejidad técnica de la transfusión; el intercambio está sometido a unas restricciones incontrolables fuera de la práctica biomédica, que decide a quién se da y quién ha de dar, especialmente, con el descubrimiento del riesgo de contagio de enfermedades como la hepatitis o el virus VIH, entre otros. 
La cuestión es esclarecer la dificultad del dar y del contraprestar. Para esto hay que descubrir lo que se da y estar segura de las implicaciones que tiene - ¿cuáles son las formas de solidaridad que están mediatizadas por la sangre compartida?-, de su papel en la constitución de las identidades -individual y colectiva - y de la vigencia de las alianzas basadas en un lazo de sangre. Dependiendo de cómo la sangre se considere, las implicaciones en la contraprestación son diferentes: establecimiento de compromiso o no, momento y forma... Recordemos además que el protocolo de transfusión garantiza también el anonimato de ambas partes, en el que la posibilidad de contacto personal entre receptora y donante es ilusorio, y en el que, como mucho, existe un agradecimiento personal simulado desde el banco de sangre que sirve para argumentar a favor del donar. Quien recibe solamente está en contacto con una bolsa de sangre facilitada por el CTG al centro sanitario, perteneciendo lo dado a una tercera persona desconocida. Ahí donde está presente la patrimonialización de lo fisiológico, la confianza en los servicios médicos es fundamental.

Donar es un acto anónimo en el que no hay reglas de reciprocidad; quien da, lo hace sin sellar ninguna alianza, o bajo la incertidumbre de no saber con quién lo hizo, pero contrae el compromiso moral de volver a donar (el interés prioritario de los bancos de sangre hoy en día es tanto la captación de nuevas donantes como la ritualización de las ya existentes) y en la responsabilización contractual. No hay consenso, solamente aceptación a través de un compromiso escrito por parte de las donantes de las condiciones de la donación y responsabilización de la calidad de lo donado. Por su parte, la institución se ocupa de, como mínimo, garantizar el anonimato de la donante, la no obtención de lucro con la sangre dada en gratuidad y las condiciones higiénicas apropiadas en el proceso de transfusión que eviten riesgos para la donante.

La alianza potencialmente generada emerge en una situación mal definida. No se sabe qué se da, ni quién recibe; el intercambio está mediatizado por una tercera actante bajo unas condiciones impuestas; a esto hay que añadir el peso que tal vez juegue la novedad del intercambio biomédico de organismos y fluidos en nuestra experiencia, que lleva a problematizar la identidad biológica y moral del individuo al alterar sus límites biológicos y sociales.

Para despejar esto, es básico tratar cómo se entienden las alianzas y las comunidades en referencia a la reciprocidad y, en consecuencia, la relación del individuo con respecto a las implicaciones adquiridas para sí o para los demás miembros de una comunidad. En la transfusión actual queda la relación entre humanos suplantada por una organización. Quien dona, no dona a nadie y dona potencialmente a todo lo mundo sin esperar una contraprestación directa, y de la misma manera, ocurre con quien recibe. Extraña alianza a la que todo el mundo accede en caso de necesidad, sin haber antes dado y sin compromiso de contraprestación; por lo tanto, es una alianza sin validez; la gratitud que se nos muestra pierde valor al no sellar un pacto, está vacía de contenido de reciprocidad y solamente consolida 
RIS

REVISTA INTERNACIONAL DE SOCIOLOGIA

№ 34, Enero-Abril, 2003

DAVID CASADO NEIRA

el compromiso hacia el banco de sangre. Tenemos la certeza de que se nos va a restituir cuando tengamos necesidad (obtenemos un equivalente a nuestra sangre) pero hay la misma posibilidad de obtenerlo que quien no dio antes (gorronas o freeriders). La institución aparece como responsable última del abastecimiento de sangre, no las personas implicadas, lo que anula la demanda de compromiso recíproco.

Tal como se pone de manifiesto recurrentemente entre diversas informantes, la donación anónima permite una mayor circulación indiscriminada de la sangre y una mayor disponibilidad temporal, pero también formula un problema: la prioridad de la familia o de alguien con quien hay un vínculo establecido a través de un nexo común previo como razón fundamental para donar, como muchas veces se alega; incluso se apela a la familia bajo una justificación de índole biológico, acudiendo a la legitimación biológica, tal como hacía el informante 4, quien donaría "en principio, para una necesidad familiar que es más probable que tenga su propio $\mathrm{Rh}$ ". La práctica es diferente dependiendo de si quien le demanda ayuda es un sujeto anónimo (ausencia de vínculo directo) o si quien la demanda es un sujeto conocido, insertado en una alianza establecida anteriormente y fundamentada en otras bases. En este último caso, cualquier persona vinculada actúa como potencial donante, por la obligación moral de seguir actualizando el pacto e, independientemente de la confianza depositada en el sistema biomédico, la ayuda se hará efectiva. En esta hipotética situación, la donante podría controlar las implicaciones de su acto al conocer el destino y el compromiso tácitamente adoptado. Aunque, en un contexto de donación voluntaria, no remunerada y universal, esto no se puede dar, esta lógica persiste. Un caso muy significativo es cuando la donante, al no poder donar directamente para su pariente $o$ amiga, va al día siguiente a donar, a sabiendas de que la sangre no va ir destinada a ella; con ello trata de compensar el consumo de hemoderivados que provocó, en un gesto de agradecimiento en nombre de la enferma, con lo que se pretende desplazar el compromiso anónimo hacia la comunidad de reciprocidad familiar o como una forma de restitución inmediata con el banco de sangre y cerrar el ciclo de contraprestación.

\section{CONCLUSIONES}

De diferente forma, las implicaciones de la donación como intercambio nos pueden ayudar a esclarecer determinados aspectos problemáticos de la donación. Como intercambio, la donación de sangre entra en la lógica del don, conforme a la teoría del don de Mauss, es decir, atendiendo a las obligaciones de dar, recibir y devolver. Esto nos pone en la pista de que, aunque hay otras implicaciones que quedan por tratar, la donación de sangre se convierte en un tipo muy particular de intercambio. Se hace de humano a humano, pero mediatizado por una tercera persona (jurídica: la institución) que intercede en todo el proceso en diferentes 
niveles (cómo se da, el tipo de alianza que se establece, la percepción de la sangre); se pide por necesidad, se da a voluntad, sin tener ninguna certeza de lo que se recibe, de si va ser y cómo va a ser devuelto; a lo que se suma la existencia de un contrato (un documento de conformidad) en el que se especifican las condiciones de la donación y que responsabiliza a la donante del acto hacia el banco de sangre.

Si bien parece que Mauss pecaba de optimismo con respecto a las posibilidades de la vigencia del don en las sociedades modernas como un mecanismo de redistribución de la riqueza (Godelier, 1998: 11-21, 95-99), la teoría del don, como constructo teórico, permite aproximarnos no solamente a la vigencia de la lógica del don en el dispositivo de la reciprocidad, sino que también ilumina otros aspectos de la microfísica del poder en la transfusión; además de lo obtenido, entran en juego distintas relaciones de fuerza articuladas por la gratitud y las plusvalías morales generadas: alianza, confianza, prestigio y placer. La dificultad de la donación de sangre como don nos pone sobre la mesa la confusa pervivencia de la lógica del don, de la existencia de ésta como principio regulador de todo sistema social también en contextos en los que se institucionalizan y despersonalizan las alianzas sociales. El don se convierte en un recurso lateral ahí donde el sistema de prestaciones totales se sustituye por instituciones que externalizan la ley y el consenso; el don, como base de la ordenación social, diluye su fuerza para convertirse en un instrumento subsidiario de la articulación social. La diferencia no estriba entre las virtudes del don comunitario y el peligro de desestructuración y egoísmo derivados del don interpersonal, como normalmente se interpreta, sino en la vigencia confusa de la lógica del don y en la necesaria suplantación provocada por las técnicas de transfusión.

La despersonalización de la sangre, que pasa a ser un producto, debilita su papel de vinculador de reciprocidad al ser algo intranscendente en la estructuración social. La sangre transfusionada es medicamentalizada; de hecho, lo que se utilizará después será un hemoderivado: sangre tratada, fraccionada y procedente de varias donantes diferentes en una misma unidad de conservación. La implantación de la concepción corporal biomedicalizada o civilizada, se plasma en prácticas y enunciaciones sobre la construcción y percepción del cuerpo - y de la sangre - y en la confianza depositada en la institución biomédica; ambos, requisitos imprescindibles para hacer factible la donación voluntaria, no remunerada y universal. 


\section{BIBLIOGRAFÍA}

ABRIL, G. (1986), “La palabra y la dádiva”, Revista de Occidente, n 67, pp. 65-78.

BENVENISTE, É. (1983), Vocabulario de las instituciones indoeuropeas, Madrid, Taurus.

BERNARD, J. (1998), O sangue, Lisboa, Instituto Piaget.

BORRILLO, D. (1994), "Estatuto y representación del cuerpo humano en el sistema jurídico", $R E I S, \mathrm{n}^{\circ} 68$, pp. 211-222.

CARO BAROJA, J. (1986), “Dones y regalos”, Revista de Occidente, n 67, pp. 5-10.

DOUGLAS, M. (1991), Pureza y peligro, Madrid, Siglo XXI.

EDWARDS, P. W. y A. ZEICHNER (1985), "Blood donor development: effects of personality, motivational and situational variables", Personality and Individual Differences, n 6, pp. 743-751.

EKEH, P.P. (1974), Social Exchange Theory: The Two Traditions, Cambridge (Massachusetts), Harvard Universiry Press.

FERRO RUIBAL, X. (1987), Refraneiro galego popular, Vigo, Editorial Galaxia.

FIRTH, R. (1974), Temas de antropologia económica, México, Fondo de Cultura Económica.

GODELIER, M. (1998), El enigma del don, Barcelona, Paidós.

GONDAR PORTASANY, M. (1993), Crítica da razón galega, Vigo, Promocións Culturais Galegas.

INRA-Europe (1995), Eurobarometer 41.0. Europeans and Blood, Brussels, European Commision.

LEMENNICIER, B. (1991), “Le corps humain: propriété de l'état ou propriété de soi?", Droits, no 13, pp. 111-122.

LÉVI-STRAUSS, C. (1983), Las estructuras elementales del parentesco, México, Fondo de Cultura Económica.

(1991), "Introducción a la obra de Marcel Mauss", en M. Mauss, Sociología y antropología, Madrid, Tecnos, pp. 13-42.

MALINOWSKI, B. (1973), Los argonautas del Pacifico occidental, Barcelona, Península.

MAUSS, M. (1991), "Ensayo sobre los dones. Motivo y forma del cambio en las sociedades primitivas", Sociologia y antropologia, Madrid, Editorial Tecnos, pp. 153-263.

MOL, A. y J. LAW (1994), "Regions, Networks and Fluids: Anaemia and Social Topology", Social Studies of Science, vol. 24, pp. 641-671.

MOLINER, M. (1998), Diccionario de uso del español, Madrid, Editorial Gredos. 
PILIAVIN, J.A. (1990), "Why do they Give the Gift of Life? A Review of Research on Blood Donors Since 1977", Transfusion, vol. 30, n5, pp. 444-459.

PILIAVIN, J. A. y P. L. CALLERO (2000), Giving the Gift of Life to Unnamed Strangers: the American Community Responsibility Blood Donor, Baltimore, Johns Hopkins University Press.

RADCLIFFE-BROWN, A. (1922), Adaman Islanders, Cambridge, Cambridge University Press.

RODRÍGUEZ VILLANUEVA, J. (1994), "Necesidades de marketing en la gestión de donantes de sangre", en J. Rodríguez Villanueva (ed.), Planificación de la hemodonación, Madrid, Sociedad Española de Tranfusión Sanguínea, pp. 19-50.

SACKS, O. (1998), Con una sola pierna, Barcelona, Editorial Anagrama.

STARR, D. (2000), Historia de la sangre, Barcelona, Ediciones B.

TITMUSS, R.M. (1970), The Gift Relationship: Fron Human Blood to Social Policy, London, Allen \& Unwin.

TURNER, B.S. (1992), Regulating Bodies. Essays in Medical Sociology, Londres-Nueva York, Routledge. 